between individuals of the same neighborhood. Results: A limited activity space modifies the association between neighborhood socioeconomic status and dementia incidence. In people whose activity space was limited to their neighborhood $(n=772,11 \%)$, living in a deprived neighborhood was associated with a higher risk of dementia (3C deprivation score: $\mathrm{T} 3 \mathrm{HR}=1.45,95 \% \mathrm{CI} 1.01-2.06$ ); conversely living in an advantaged neighborhood was associated with a lower risk (Table 1). These results were not evidenced for people whose activity space was not limited. Conclusions: This study shows the role of activity space in health inequalities, in addition to highlighting the importance of the living environment on cognitive ageing. If confirmed in different populations, these findings would help to identify groups at risk, best target for prevention. 1. Letellier $\mathrm{N}$ et al. (2017). Sex-specific association between neighborhood characteristics and dementia: The Three-City cohort. Alzheimers Dement.

\section{2-10-06 BIOLOGICAL MECHANISMS UNDERLYING THE LONGITUDINAL EFFECTS OF SOCIAL STRESS ON AD RISK}

Laura B. Zahodne, University of Michigan, Ann Arbor, MI, USA. Contact e-mail: lzahodne@umich.edu

Background:Previous research suggests that social stress may be an independent risk factor for Alzheimer's disease (AD), which may contribute to inequalities across sociodemographic groups. The biological mechanisms underlying the link between social stress and AD are unclear but may involve inflammatory processes. This study aimed to determine whether systemic inflammation, indexed by blood levels of C-reactive protein (CRP), mediates associations between perceived discrimination and episodic memory trajectories over four years. Methods: Using longitudinal cognitive and biomarker data from 11,470 participants aged 50 and older in the Health and Retirement Study, structural equation models quantified associations between baseline perceived discrimination and fouryear trajectories of CRP and episodic memory, controlling for sociodemographic characteristics, smoking status, and chronic disease burden. Results: Higher perceived discrimination was associated with lower baseline memory and faster memory decline. Higher CRP at baseline partially mediated the negative association between discrimination and baseline memory, but CRP trajectories did not mediate the association between discrimination and memory decline. Conclusions: This U.S.representative longitudinal study provides evidence for deleterious effects of discrimination on memory aging, a key indicator of $\mathrm{AD}$ risk. The fact that elevated CRP only partially explained the harmful effects of discrimination in this study highlights the need for more comprehensive investigations of biological mechanisms underlying the apparent link between social stress and AD risk in order to better characterize potential intervention targets.

\section{ORAL SESSIONS}

02-11

DEVELOPMENT OF NEW MODELS AND ANALYSIS METHODS: INFLAMMATION - A MARKER OF DISEASE SEVERITY

\section{O2-11-01 WITHDRAWN}

O2-11-02 THE ROLE OF SOLUBLE TNF IN METABOLIC DYSFUNCTION AND BBB ALTERATIONS IN A MOUSE MODEL OF ALZHEIMER'S DISEASE

Malu G. Tansey, Kathryn P. MacPherson, Lori N. Eidson, Mary K. Herrick, Maria Elizabeth de Sousa Rodrigues, Danielle Oliver, Sean D. Kelly, Yuan Yang, Jianjun Chang, Lindsey Sniffen, Emory University School of Medicine, Atlanta, GA, USA.Contacte-mail:malu.tansey@emory.edu

Background:Peripheral immune cell signaling has been shown to play an important role in neuroinflammatory diseases, such as Alzheimer's disease (AD). Cytokine and chemokine mechanisms regulate peripheral immune cell trafficking to inflamed tissues, such as the brain. The cytokine, soluble Tumor Necrosis Factor (solTNF), has been shown to be elevated in AD patients. SolTNF regulates blood-brain barrier permeability, and is produced by central and peripheral immune cells. Therefore, we hypothesize that STNF is a key mediator of peripheral immune cell contributions to AD-like pathology. Peripheral inflammation may accelerate neurodegeneration by increasing neuroinflammation, altering blood brain barrier integrity, and promoting peripheral immune cell trafficking to the CNS. Therefore, we aim to determine the effect of chronic high-fat high-carbohydrate (HFHC) diet-induced peripheral inflammation on neuroinflammation and neuronal health in a model of AD. Methods: The $5 x F A D$ mouse model of AD was fed a high-fat high-carbohydrate (HFHC) or a control diet (CD) for 8 weeks. After 4 weeks of diet, XPro ${ }^{\circledR 1595}$, a BBB-permeant peptide, was used to selectively inhibit solTNF signaling. Tight junction proteins and central inflammatory gene expression were evaluated by qPCR. Immune cell populations in the brain were assessed using flow cytometry. Results: 5xFAD mice fed a HFHC diet and treated with saline had increased TNF mRNA expression in the hippocampus. XPro ${ }^{\circledR} 1595$ eliminated this diet effect. Independent of genotype, mice given HFHC diet and saline had increased hippocampal ZO-1 gene expression compared to $\mathrm{CD}$-fed mice given saline. XPro ${ }^{\circledR} 1595$ reversed this diet effect. HFHC diet treatment also impacted brain immune populations. 5xFAD mice had increased CD8+ $\mathrm{T}$ cell percentages in the brain when fed HFHC. CD8+ T cells were also increased in HFHC diet-fed Tg mice as compared with $\mathrm{HFHC}$ nonTg mice. An increase in Ly6C $+\mathrm{T}$ cells was observed in HFHC diet-fed Tg mice versus $\mathrm{CD}$ fed mice, suggesting an increased migration of CD8+ (cytotoxic) $\mathrm{T}$ cells into the brain. Experiments are ongoing to assess additional effects of solTNF in AD pathogenesis. Conclusions: Diet-induced obesity alters immune cell populations and promotes BBB-associated alterations that may impact neuroinflammation and increase the risk for AD; targeting solTNF may be a therapeutic path forward.

\section{O2-11-03 PROGRANULIN LOSS DYSREGULATES SPLENIC AND PERIPHERAL BLOOD IMMUNE CELL POPULATIONS AND MAY CONTRIBUTE TO NEUROINFLAMMATION AND NEURODEGENERATION IN FRONTOTEMPORAL DEMENTIA}

Thomas G. Kukar, Kathryn P. MacPherson, George T. Kannarkat, Elizabeth M. Kline, Christopher M. Holler, Michelle A. Johnson, 